\title{
Analytical methods for prefiltering of close approaches between space objects
}

\author{
M. Xavier James Raj* and M. Mutyalarao \\ Applied Mathematics Division, Vikram Sarabhai Space Centre, Trivandrum - 695 022, INDIA \\ "Corresponding Authors: e-mails: m_xavierjamesraj@vssc.gov.in (Xavier James Raj), m_mutyalarao@yahoo.co.in (M. Mutyalarao)
}

\begin{abstract}
In the history of space, for the first time an operational US communications satellite Iridium 33, in low-Earth orbit was struck and destroyed by the collision of a long defunct Russian communications satellite COSMOS 2251 on $10^{\text {th }}$ February 2010. It is necessary to understand the circumstances of this collision for avoiding similar incidents in the future. In this direction a detailed conjunction analysis of all space objects is essential. Since a large number of objects are orbiting in space, it is very difficult to find out the close approach for all objects with simulations. Hence, a study related to the prefiltering of close approaches between space objects using analytical techniques is carried out in this paper. It is found that the presented methodology with prefiltering techniques is effective in reducing the number of objects for simulations.
\end{abstract}

Keywords: Collision, orbit, prefiltering, debris, operational satellite.

DOI: http://dx.doi.org/10.4314/ijest.v5i3.4

\section{Introduction}

Due to a high increase in the number of objects in space, like satellites, pay loads, mission related objects, debris like rocket bodies, spent stages, etc., prediction of close approaches between orbiting objects is an important factor to avoid collision between the operational satellite and other orbiting objects. To protect an operational satellite, called 'primary' in this paper, from colliding with another space object, called 'secondary' here, their orbits must be computed simultaneously and the distance between them should be monitored. Recently, space scientists all over the Globe are giving much importance to the problem of collision avoidances (Alarcon-Rodriguez et al., 2004, Gronchi, 2005 and Choi et al., 2009) for the stability of future Low Earth Orbit (LEO) environment to protect the launch vehicles and operational satellites from the collision.

In this paper, a detailed study related to the prefiltering of close approaches between space objects using analytical techniques (Hoots et al., 1984) are carried out. The analytical equations are solved using an iterative technique. The prefiltering solutions are obtained in an efficient manner, which eliminates many objects from considering long term orbit simulations for close approaches. The methodology is valid for all values of eccentricities less than one and all relative geometries between the two orbits. As a test case, we have utilized a data base consisting of 23544 simulated LEO-crossing objects of size greater than or equal to $10 \mathrm{~cm}$. Using these analytical methods more than $98 \%$ of the objects were prefiltered. Only for the remaining $2 \%$ of the objects, the close approaches using the long term orbit predictions are necessary. As a result, these analytical methods are much more efficient in reducing the total number of simulations which need to be carried out for further analysis.

In general, the problem is stated by specifying a time span $\left[T_{b}, T_{e}\right]$, and a separation distance $D$ between two objects moves around their respective orbits in space, the relative separation distance between the two orbiting bodies is

$$
r_{\text {rel }}=\left|\bar{r}_{p}-\bar{r}_{s}\right|,
$$


where $\bar{r}$ is the radius vector in the Earth centered frame and the subscripts $p$ and $s$ stand for primary and secondary objects, respectively, over the span of time interest $\left[\mathrm{T}_{\mathrm{b}}, \mathrm{T}_{\mathrm{e}}\right]$. Figure 1 shows a typical graph of relative distance $r_{\text {rel }}$.

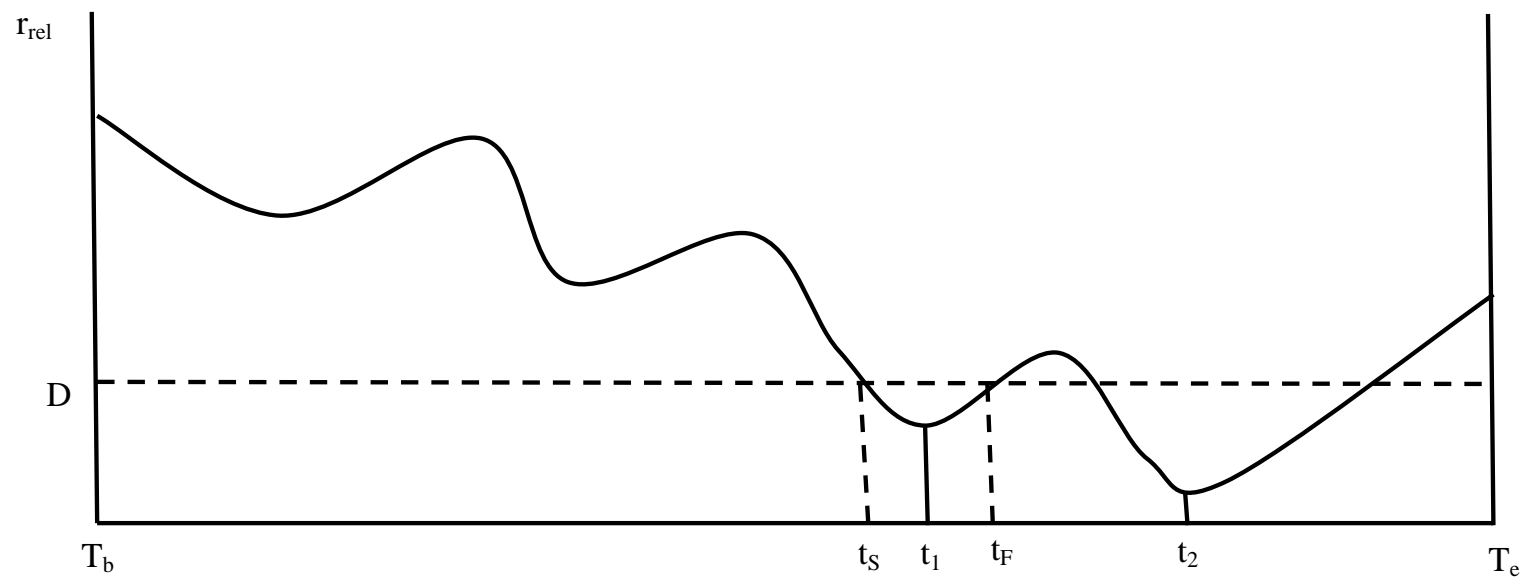

Figure 1: Relative separation distance.

Since these points are simply relative minima of the function $r_{\text {rel }}$, the most obvious solution to the problem would be a numerical technique. Starting at the beginning of the time interval of interest $\mathrm{T}_{\mathrm{b}}$, we could numerically step along the primary and secondary orbits noting the relative separation distances. Whenever the relative distance changed from decreasing to increasing, we would know that the two objects had passed relative minima. If these minima were less than D, we would record this time as a time of close approach. Stepping would continue until we reached the end of the time interval of interest $\mathrm{T}_{\mathrm{e}}$. If we wanted to find all times of close approach with all orbiting objects, the procedure would be repeated for each of the total secondaries. Clearly, this procedure consumes exorbitant amounts of computer processing time with large number of simulations. Hence, when we consider the task of collision alerting for many primaries, use of numerical techniques becomes hopeless.

Thus, to make the task manageable, we consider two analytical approaches methods for determining the close approaches between two orbiting bodies.

\section{Perigee-Apogee prefilter}

Many of the possible secondaries can be eliminated from consideration, because of the relative geometry of the two ellipses, which does not allow the two trajectories to ever come within the threshold distance $D$. The simplest test is perigee-apogee test. Let q denote the larger of the two perigees and Q denote the smaller of the two apogees. If

$$
q-Q>D
$$

then the secondary will not come within the distance $D$ and it need not be considered for further analysis/simulation.

\section{Geometrical prefilter}

This prefilter considers the relative geometry of two ellipses in space. Figure 2 illustrates the two arbitrary ellipses and the line intersection of the orbital planes. There will be two closest approach distance $d_{1}$ and $d_{2}$ between the two elliptical paths. It should be noted that the points are not necessarily along the line of intersection of the two planes. 


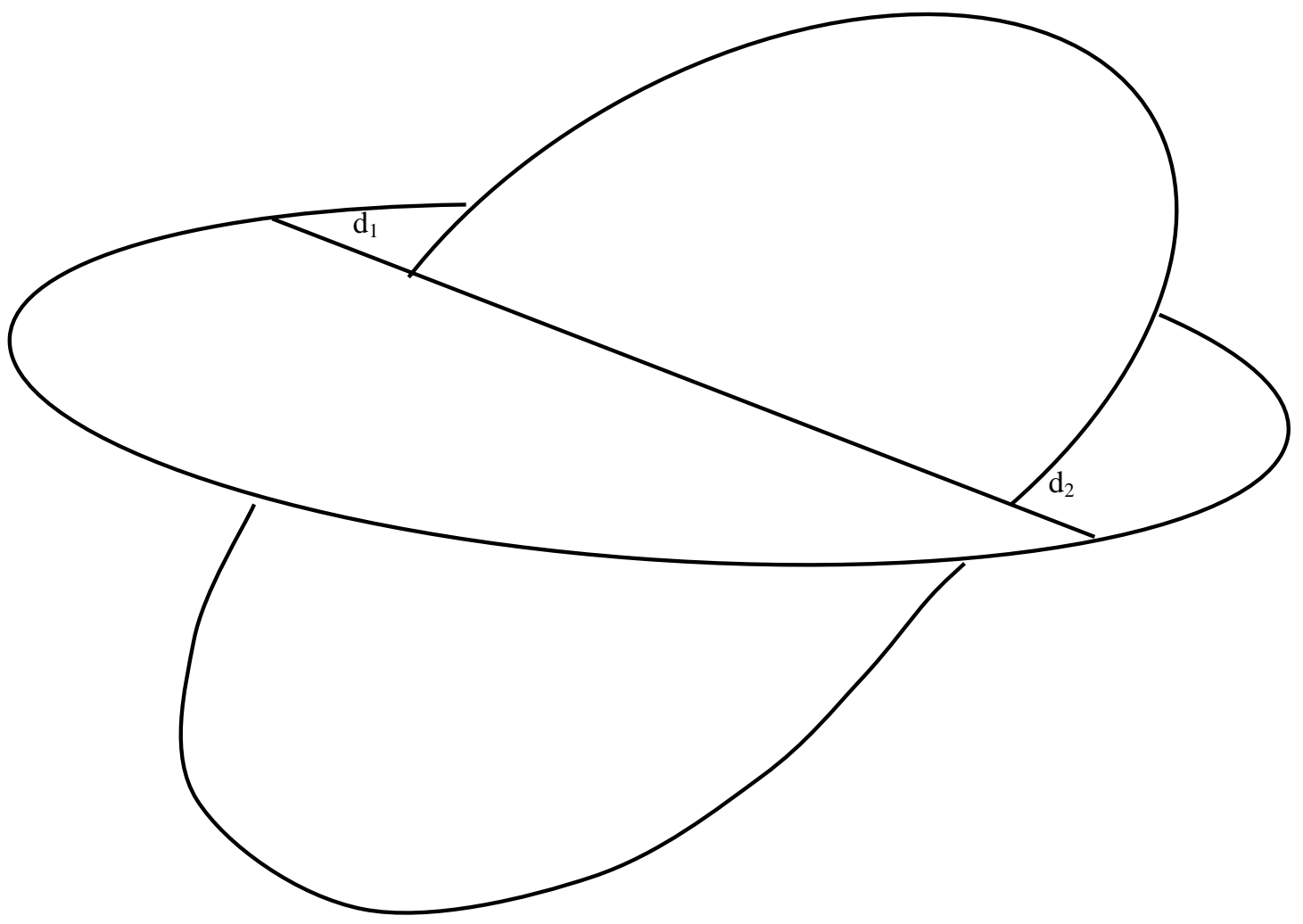

Figure 2: Geometrical prefilter.

This is a purely geometrical calculation and is independent of the location of the two satellites on their paths. If the minimum distance in the space between the two paths is greater than the separation distance $\mathrm{D}$, then the satellites cannot have a close encounter of interest. Let

$$
\bar{K}=\bar{w}_{s} \times \bar{w}_{p},
$$

where $\bar{w}_{s}$ and $\bar{w}_{p}$ are unit vectors normal to the orbital planes of secondary and primary objects, respectively. These unit vectors can be calculated using the expression

$$
\bar{W}=(\sin \Omega \sin I) \boldsymbol{i}+(\cos \Omega \sin I) \boldsymbol{j}+(\cos I) \boldsymbol{k}
$$

where $\Omega$ is the right ascension of the ascending node (RAAN), $I$ is the inclination of the orbit and $\boldsymbol{i}, \boldsymbol{j}$ and $\boldsymbol{k}$ are unit vectors along $\mathrm{x}, \mathrm{y}$ and $\mathrm{z}$ directions.

The vector $\bar{K}$ lies along the line of intersection of the two planes and has the property that

$$
|\bar{K}|=\sin I_{R} \text {, }
$$

where, $I_{R}$ is the relative inclination angle between the two orbital planes. It should be noted that, $I_{R}$ is not simply the difference between the inclinations of the two orbits. If $I_{R}$ is identically zero, then the geometrical prefilter equations become indeterminate. The orientation of the vector $\bar{K}$ within the orbital plane of the either satellite is given by the angle $\Delta$ between the line of ascending node of the satellite plane and the vector $\bar{K}$ as illustrated in Figure 3. Let

$$
\bar{n}_{p}=\cos \Omega_{\rho} \boldsymbol{i}+\sin \Omega_{\rho} \boldsymbol{j}
$$

be a unit vector along the line of ascending node of the primary satellite. Then

$$
\bar{K} \cdot \bar{n}_{p}=\sin I_{p} \cos I_{s}-\sin I_{s} \cos I_{p} \cos \left(\Omega_{p}-\Omega_{s}\right)
$$

But from Figure 3, it is clear that

$$
\bar{K} \cdot \bar{n}_{p}=\bar{K} \cos \Delta_{p}
$$




$$
=\sin I_{R} \cos \Delta_{p}
$$

From Eqs. (6) and (7), we obtain

$$
\cos \Delta_{p}=\left[\sin I_{p} \cos I_{s}-\sin I_{s} \cos I_{p} \cos \left(\Omega_{p}-\Omega_{s}\right)\right] / \sin I_{R}
$$

Also, by using the law of sines in spherical trigonometry, we get

$$
\sin \Delta_{p}=\left[\sin I_{s} \sin \left(\Omega_{p}-\Omega_{s}\right)\right] / \sin I_{R}
$$

From the above two equations, $\Delta_{\mathrm{p}}$ is uniquely determined. Similarly, it can be shown that

$$
\cos \Delta_{s}=\left[\sin I_{p} \cos I_{s} \cos \left(\Omega_{p}-\Omega_{s}\right)-\sin I_{s} \cos I_{p}\right] / \sin I_{R}
$$

and

$$
\sin \Delta_{s}=\left[\sin I_{p} \sin \left(\Omega_{p}-\Omega_{s}\right)\right] / \sin I_{R}
$$

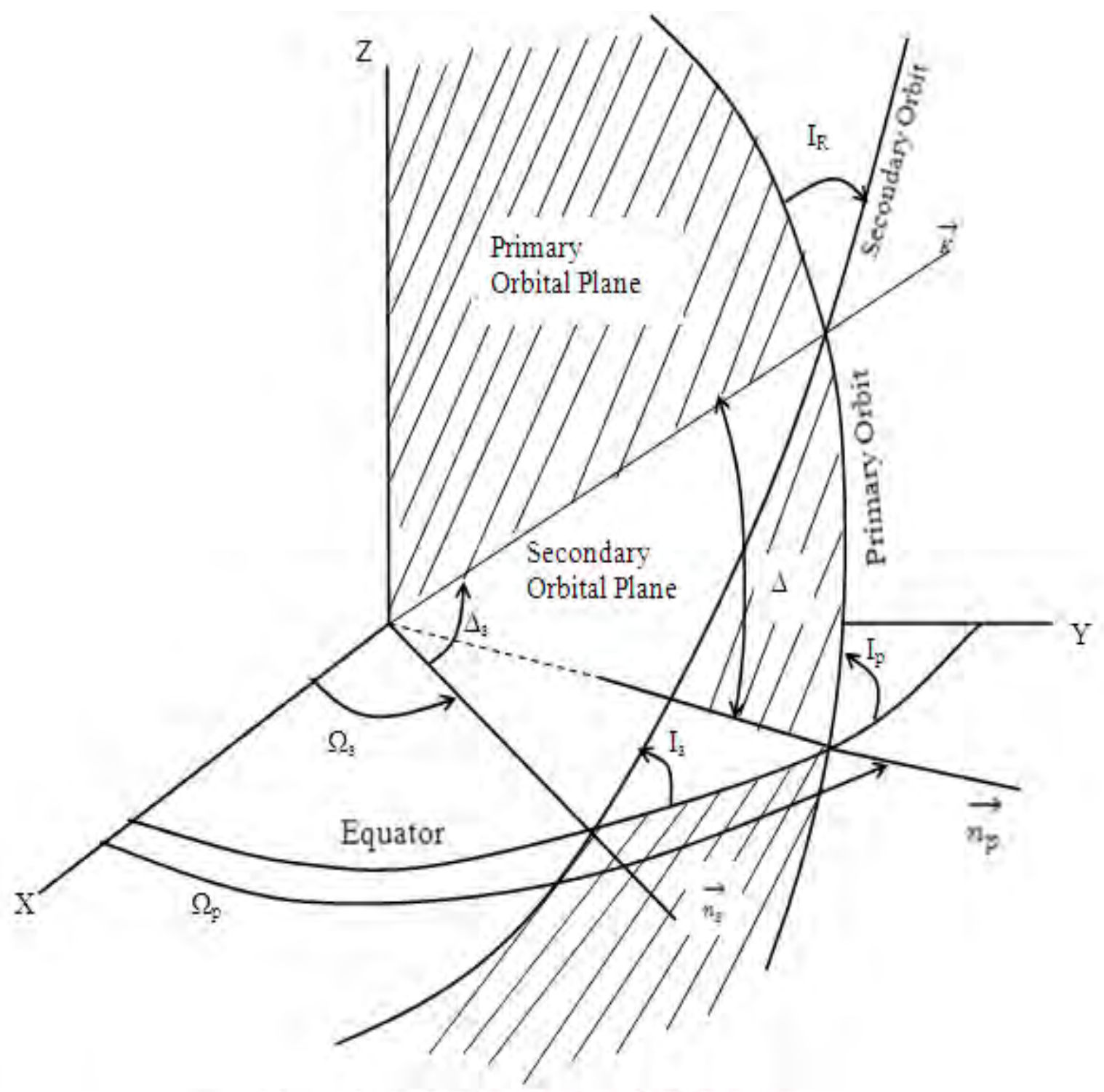

Figure 3: Relative orientation of orbital planes. 
Let $U_{R}$ denote the angle within the orbital plane from the vector $\bar{K}$ to the satellite position measured positive in the sense of the satellite motion as shown in Figure 4.

Let $r_{p}$ and $r_{s}$ denote the magnitude of the position vectors of the primary and secondary satellites and let $\gamma$ denote the angle between the two position vectors. Then

$$
r_{r e l}^{2}=r_{p}^{2}+r_{s}^{2}-2 r_{p} r_{s} \cos \gamma
$$

From spherical trigonometry we get,

$$
\cos \gamma=\cos u_{R_{P}} \cos u_{R_{P}}+\sin u_{R_{P}} \sin u_{R_{P}} \cos I_{R}
$$

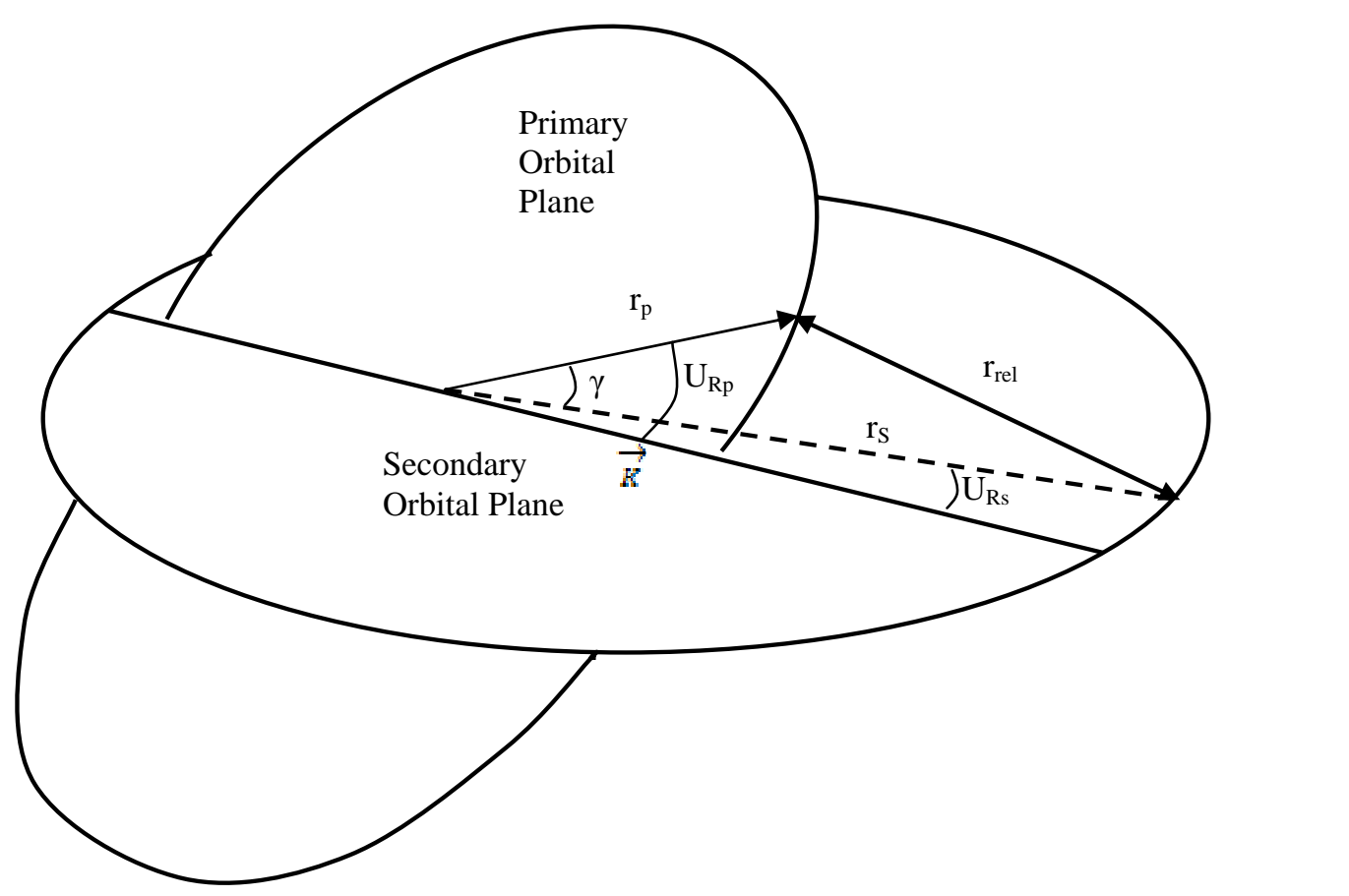

Figure 4: Satellite positions in orbital planes.

The point of closest approach of the elliptical paths will be a relative minimum of the function in Eq. (12). It is a function of the true anomalies of the two satellites since

$$
u_{R}=\mathrm{f}+\omega-\Delta
$$

where $\mathrm{f}$ is the true anomaly and $\omega$ is the argument of perigee of the orbit.

Thus, for the relative minimum the solution of following two simultaneous equations need to be solved

$$
\frac{\partial r_{r e l}^{2}}{\partial r_{p}}=0 \quad \text { and } \quad \frac{\partial r_{r e l}^{2}}{\partial r_{s}}=0
$$

The above Eq. (15) is simplified using Eqs. (13) and (14) as follows

$$
\begin{aligned}
& r_{p} e_{p} \sin f_{p}+r_{s}\left[\cos u_{R_{s}}\left(\sin u_{R_{P}}+a_{y_{p}}\right)-\sin u_{R_{P}} \cos I_{R}\left(\cos u_{R_{p}}+a_{x_{p}}\right)\right]=0 \\
& r_{s} e_{s} \sin f_{s}+r_{p}\left[\cos u_{R_{P}}\left(\sin u_{R_{P}}+a_{y_{s}}\right)-\sin u_{R_{s}} \cos I_{R}\left(\cos u_{R_{s}}+a_{x_{s}}\right)\right]=0
\end{aligned}
$$

where we have introduced the notations

$$
a_{x}=e \cos (\omega-\Delta) \text { and } a_{y}=e \sin (\omega-\Delta)
$$


For the case of two near circular orbits Eqs. (16) have the solutions

$$
f_{p}=\Delta_{p}-\omega_{p} \text { and } f_{s}=\Delta_{s}-\omega_{s}
$$

For noncircular cases the equations readily admit an iterative solution by Newton`s method (Conte and De Boor, 2000). Given equations are of the form

$$
F\left(f_{p}, f_{s}\right)=0 \text { and } G\left(f_{p}, f_{s}\right)=0,
$$

Newton's method gives the iterative solution

where

$$
f_{p_{i+1}}=f_{p_{i}}+h \text { and } f_{s_{i+1}}=f_{s_{i}}+k
$$

$$
\begin{aligned}
& h=\frac{F \frac{\partial G}{\partial f_{s}}-G \frac{\partial F}{\partial f_{s}}}{\frac{\partial F}{\partial f_{s}} \frac{\partial G}{\partial f_{p}}-\frac{\partial F}{\partial f_{p}} \frac{\partial G}{\partial f_{s}}} \\
& k=\frac{G \frac{\partial F}{\partial f_{p}}-F \frac{\partial G}{\partial f_{p}}}{\frac{\partial F}{\partial f_{s}} \frac{\partial G}{\partial f_{p}}-\frac{\partial F}{\partial f_{p}} \frac{\partial G}{\partial f_{s}}} \\
& F=r_{p} e_{p} \sin f_{p}+r_{s}\left(A \cos u_{R_{p}}-B \cos I_{R} \sin u_{R_{p}}\right) \\
& G=r_{s} e_{s} \sin f_{s}+r_{p}\left(C \cos u_{R_{p}}-D \cos I_{R} \sin u_{R_{p}}\right) \\
& \frac{\partial F}{\partial f_{p}}=r_{p} e_{p} \cos E_{p}+r_{s} \cos \gamma \\
& \frac{\partial F}{\partial f_{s}}=-\frac{r_{s}}{1+e_{s} \cos f_{s}}\left(A C+B D \cos I_{R}\right) \\
& \frac{\partial G}{\partial f_{p}}=-\frac{r_{p}}{1+e_{p} \cos f_{p}}\left(A C+B D \cos I_{R}\right) \\
& \frac{\partial G}{\partial f_{s}}=r_{s} e_{s} \cos E_{p}+r_{p} \cos \gamma
\end{aligned}
$$

with $A=\sin u_{R_{P}}+a_{y_{p}} B=\cos u_{R_{P}}+a_{x_{p}} C=\sin u_{R_{s}}+a_{y_{s}}$ and $D=\cos u_{R_{s}}+a_{x_{s}}$

where, $E$ denotes the eccentric anomaly. A starting value for the iteration is given by Eq.(18). Let $f_{p}^{*}$ and $f_{s}^{*}$ denote the final converged values from the iteration. These are used in Eq. (12) to calculate one point of closest approach between the two elliptical paths. The another point of closeness is found by iteration on Eq. (16) with starting values $f_{p_{1}}=f_{p}^{*}+\pi$ and $f_{S_{1}}=f_{s}^{*}+\pi$.

The converged values from the iteration are used in Eq. (12) to calculate the other points. If both points are greater than D, then the secondary need not be considered further analysis. 


\section{Numerical Results}

A numerical experimentation was performed with a model consisting of 23544 LEO-crossing simulated objects. The first object with its orbital details provided in Table-1 is chosen as primary and all other 23543 objects are chosen as secondaries, whose apogee and perigee combinations are plotted in Figure 5a.

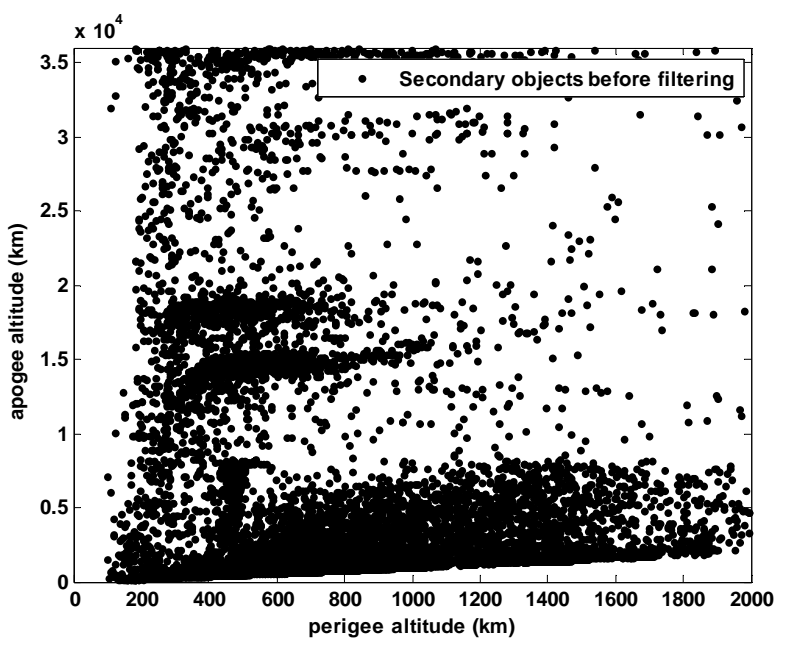

Figure 5a: Perigee/Apogee combinations of initial secondaries

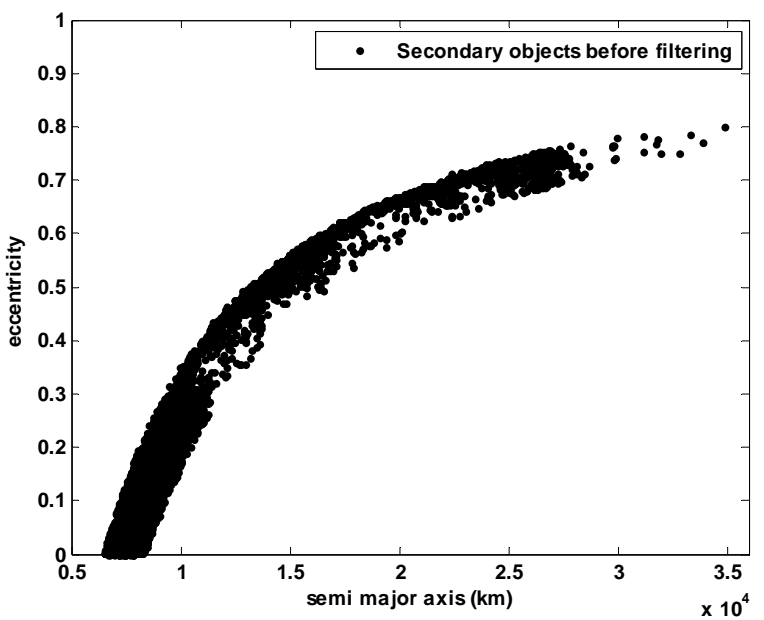

Figure 6a: Semi-major axis vs. eccentricity of initial secondaries

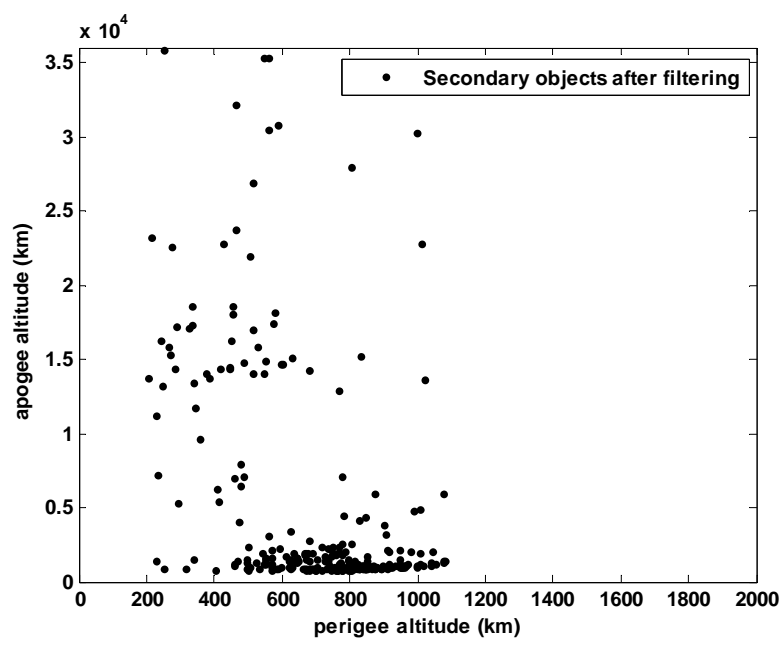

Figure 5b: Perigee/Apogee combinations of secondaries after filtering

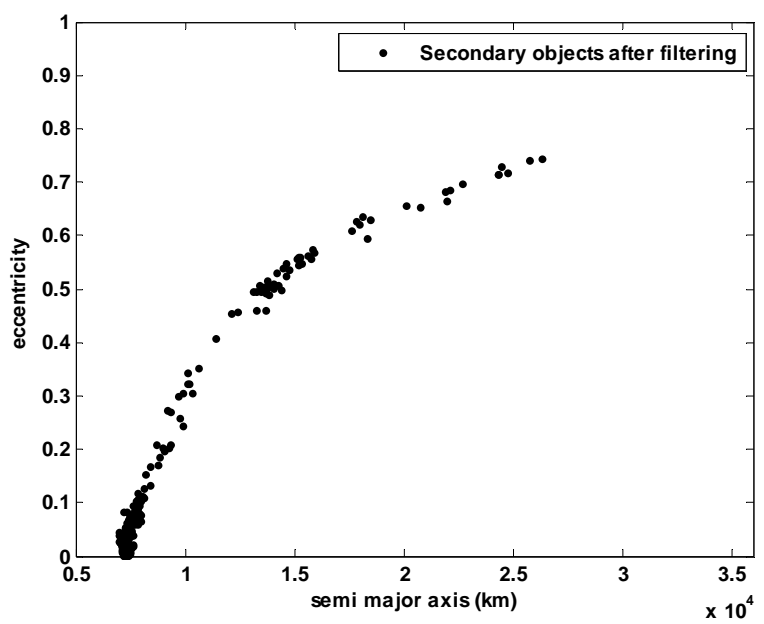

Figure 6b: Semi-major axis vs. eccentricity of secondaries after filtering 


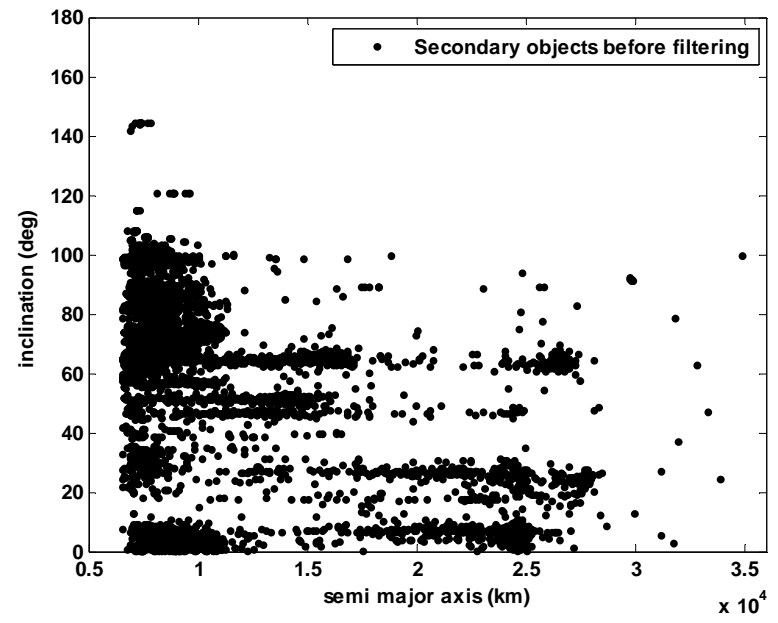

Figure 7a: Semi-major axis vs. inclination of initial secondaries

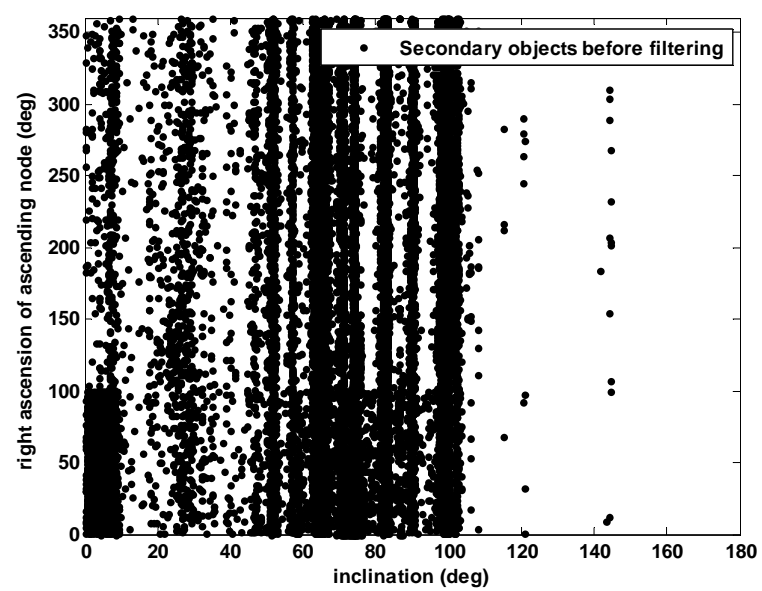

Figure 8a: Inclination vs. RAAN of initial secondaries

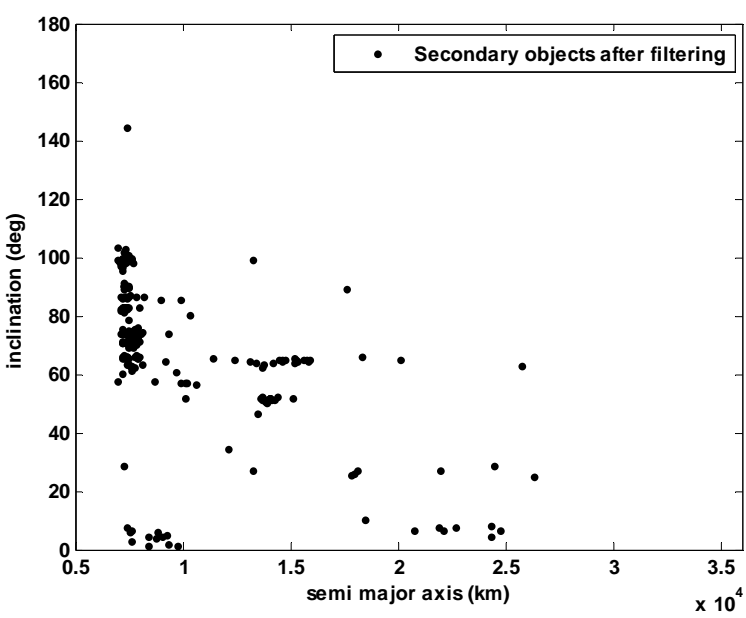

Figure 7b: Semi-major axis vs. inclination of secondaries after filtering

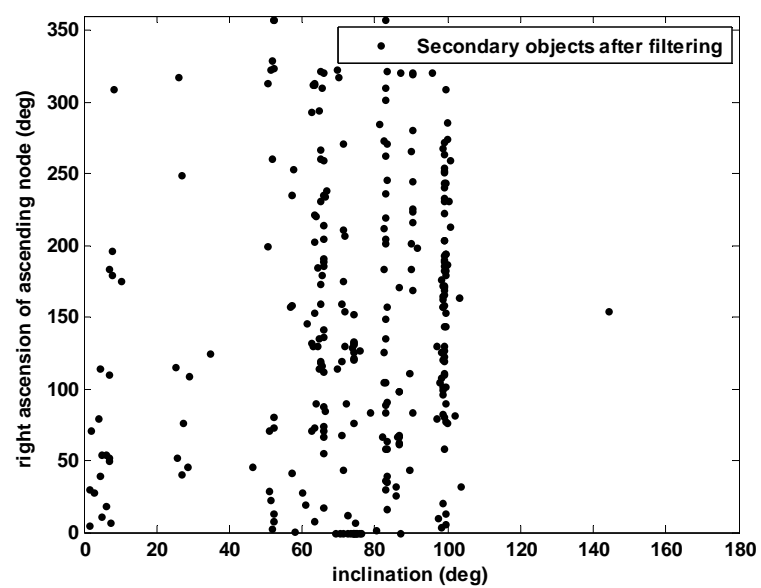

Figure 8b: Inclination vs. RAAN of secondaries after filtering

Table 1: Orbital Details of Primary Object.

\begin{tabular}{|c|c|c|c|}
\hline Semi major axis (a) & $=7325.5 \mathrm{~km}$ & Eccenticity (e) & $=0.0189$ \\
\hline Inclination (i) & $=70.94 \mathrm{deg}$ & \multicolumn{2}{|c|}{ Longitude of ascending node $(\Omega)=155.66 \mathrm{deg}$} \\
\hline \multicolumn{2}{|c|}{ Argument of perigee $(\omega)=3.57 \mathrm{deg}$} & Mean Anomaly & $=120.90 \mathrm{deg}$ \\
\hline Orbital Mass & $=2988 \mathrm{~kg}$ & Diameter & $=28.51 \mathrm{~cm}$ \\
\hline
\end{tabular}

The orbital characteristics of the 23543 secondaries such as semi-major axis vs. eccentricity, semi-major axis vs. inclination and inclination vs. right ascension of ascending node (RAAN) are plotted in Figures 6a, 7a and 8a respectively. A separation distance D of 100 meters is considered. After applying the filtering techniques presented above, the following results were obtained:

8579 objects were prefiltered by perigee-apogee prefilter and

14675 objects were prefiltered by geometrical prefilter.

So, total numbers of objects prefiltered are 23254. 
Hence, the job became much lesser with only 289 secondaries to be considered for long term analysis. So, more than $98 \%$ objects were prefiltered using these analytical methods. The effect of using these filtering techniques is presented in Figures $5 \mathrm{~b}$, $6 b, 7 b$ and $8 b$. From Figures $5 a$ and $5 b$, it is observed that the secondaries with perigee altitude of more than $1200 \mathrm{~km}$ never come within 100 meters of the primary considered in Table-1. By comparing the Figures 5a with 5b, 6a with 6b, 7a with 7b and 8a with $8 \mathrm{~b}$, one can visualize the advantage of the prefiltering techniques. Particularly, instead of propagating all objects for identifying the close approaches between the space objects one can eliminate the filtered objects and carry the simulations/analysis for the remaining few objects. As a result, these analytical methods are much more efficient with a significant amount of computer time savings due to the less number of objects for simulations.

\section{Conclusions}

In this paper, a detailed study regarding prefiltering of close approaches among space objects using analytical techniques is presented. The simulation is carried out by considering a batch of data, which includes a primary and secondaries. The study reveals that the prefiltering techniques are effective to reduce the computation of long term propagation of orbits by which collisions between space objects can be evaluated. Therefore, the paper eliminates the need for actual orbit computation for a great number of space objects for conjunction analysis.

\section{References}

Alarcon-Rodriguez J.R., Martinez-Fadrique F.M. and Klinkrad H., 2004. Development of collision risk assessment tool. Advances in Space Research. Vol 34, No. 5, pp. 1120-1124.

Gronchi G.F., 2005. An algebraic method to compute the critical points of the distance function between two Keplerian orbits. Celestial Mechanics and Dynamical Astronomy, Vol 93, pp. 295-329.

Choi Y.-K., Chiang J.W., Wang W., Kim M.S. and Elber G., 2009. Continuous collision detection for ellipsoids. IEEE Transactions on Visualization and Computer Graphics. Vol. 15, No. 2, pp. 311-325.

Hoots F.R., Crawford L. L. and Roehrich R. L., 1984. An analytical method to determine future close approaches between satellites. Celestial Mechanics. Vol. 33, pp. 143-158.

Conte S.D. and De Boor C., 2000. Elementary Numerical Analysis: An Algorithmic Approach. McGraw-Hill Higher Education.

\section{Acknowledgment}

The authors are thankful to the reviewers for their valuable comments and good suggestions, which improved the quality of the paper.

\section{Biographical notes}

M. Xavier James Raj and M. Mutyalarao are with the Applied Mathematics Division, Vikram Sarabhai Space Centre, Trivandrum - 695022 , India

Received February 2012

Accepted March 2013

Final acceptance in revised form March 2013 